\title{
The Unconventional Copper Oxide Superconductor with Conventional Constitution
}

\author{
W. M. Li $i^{1,2,3} \cdot$ J. F. Zhao ${ }^{1,2} \cdot$ L. P. Cao ${ }^{1,2} \cdot$ Z. Hu ${ }^{4} \cdot$ Q. Z. Huang ${ }^{5} \cdot$ X. C. Wang ${ }^{1,2,3} \cdot$ R. Z. Yu ${ }^{1,2,3} \cdot$ Y. W. Long ${ }^{1,2,3} \cdot$ H. Wu ${ }^{5} \cdot$

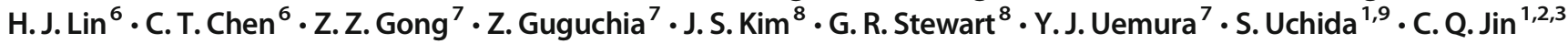

Received: 3 August 2019 / Accepted: 10 September 2019 / Published online: 30 November 2019

(C) The Author(s) 2019

\begin{abstract}
A new $\mathrm{Ba}_{2} \mathrm{CuO}_{4-y}$ superconductor with critical temperature $\left(T_{c}\right)$ exceeding $70 \mathrm{~K}$ was discovered. The $\mathrm{X}$-ray absorption measurement gives evidence that this cuprate resembles $\mathrm{La}_{2} \mathrm{CuO}_{4}$ but is doped with a fairly large amount of holes, while in contrast to the so far known hole-doped high- $T_{c}$ cuprates, the new cuprate possesses a much shorter local apical oxygen distance as well as much expanded in-plane $\mathrm{Cu}-\mathrm{O}$ bond, leading to unprecedented compressed local octahedron. In compressed local octahedron, the $\mathrm{Cu} 3 d 3 z^{2}-r^{2}$ orbital level will be lifted above the $\mathrm{Cu} 3 d x^{2}-y^{2}$ orbital level with more three-dimensional features, implying that pairing symmetry may carry admixtures from more than one gap, suggesting that $\mathrm{Ba}_{2} \mathrm{CuO}_{4-y}$ composed of alkaline earth copper oxides that are the essential elements to form cuprate superconductors may belong to a new branch of cuprate superconductors.
\end{abstract}

Keywords Unconventional cuprates $\cdot$ Compressed octahedron $\cdot$ High pressure

\section{Introduction}

Searching for superconductors with higher $T_{c}$ has attracted great interest since cuprate superconductors was discovered [1-9]. It is widely accepted that the superconducting critical temperature $\left(T_{c}\right)$ is a function of carrier doping level, i.e., $T_{c}$ becomes maximum at optimal doping while gradually

C. Q. Jin

Jin@iphy.ac.cn

1 Institute of Physics, Chinese Academy of Sciences, Beijing 100190, China

2 School of Physics, University of Chinese Academy of Sciences, Chinese Academy of Sciences, Beijing 100190, China

3 Materials Research Lab, Songshan Lake, Dongguan 523808, China

4 Max Planck Institute for Chemical Physics of Solids, Nöthnitzer Straße 40, 01187 Dresden, Germany

5 NIST Center for Neutron Research, Gaithersburg, MD 20899, USA

6 National Synchrotron Radiation Research Center, 30076 Hsinchu, Taiwan

7 Department of Physics, Columbia University, New York, NY 10027 , USA

8 Department of Physics, University of Florida, Gainesville, FL 32611, USA

9 Department of Physics, University of Tokyo, Tokyo 113-0033, Japan diminishing at both sides of underdoped or overdoped regions $[10,11]$. Consensus is currently that a $T_{c}$ dome in the low $p$ region (optimum doping around $p \sim 0.15$ ) is born out of the Mott insulator with antiferromagnetic (AF) order [12, 13], and high $T_{c}$ superconductivity (HTS) emerges from around $p \sim$ 0.08 [14]. In the overdoping region, $T_{c}$ vanishes again when the doping level is larger than a critical value $p_{\max } \sim 0.27$ [11]. The material is deemed a Fermi-liquid-like metal in which electronic correlations become weak in heavily overdoped regions. But a growing number of researches in recent years suggest that it shows not a simply Fermi liquid behavior even in very highly overdoped copper oxides $[15,16]$.

Prof. T. H. Geballe is the pioneer who paid much attention to the unusual phenomenon in the heavily doped region of cuprates $[1,17,18]$. Some cuprates synthesized at a high pressure show very high $T_{c}$ in the strongly overdoped region well beyond the normal superconducting phase diagram for the established cuprate superconductors [18-20]. This raised the question to the paradigm where the high $T_{c}$ of cuprates actually originates from [17]. One typical system is $\mathrm{Cu}_{0.75} \mathrm{Mo}_{0.25} \mathrm{Sr}_{2} \mathrm{YCu}_{2} \mathrm{O}_{7.54}$ with double $\mathrm{CuO}$ layers in the unit cell similar to $\mathrm{YBCO} 123$ phase which is in the heavily overdoped regime ( $p \sim 0.46$ ) but shows a $T_{c}$ up to $87 \mathrm{~K}[19$, 21]. Another system is a monolayer $\mathrm{CuO}_{2}$ deposited on a single crystal of $\mathrm{Bi}_{2} \mathrm{Sr}_{2} \mathrm{CaCl}_{2} \mathrm{O}_{8+\delta}$ which is thought to be heavily overdoped $(p \sim 0.9)$ due to charge transfer at the interface $[22,23]$. Besides, a very important system is $\mathrm{Sr}_{2} \mathrm{CuO}_{3+\delta}$ 
Fig. 1 Schematic crystal structure of the $\mathrm{Ba}_{2} \mathrm{CuO}_{4-y}$, that is isostructural to $\mathrm{La}_{2} \mathrm{CuO}_{4}$ but with a compressed copper oxygen octahedron (the blue ball is $\mathrm{Ba}$ and the red ball is copper, while oxygen is at the ligand site)
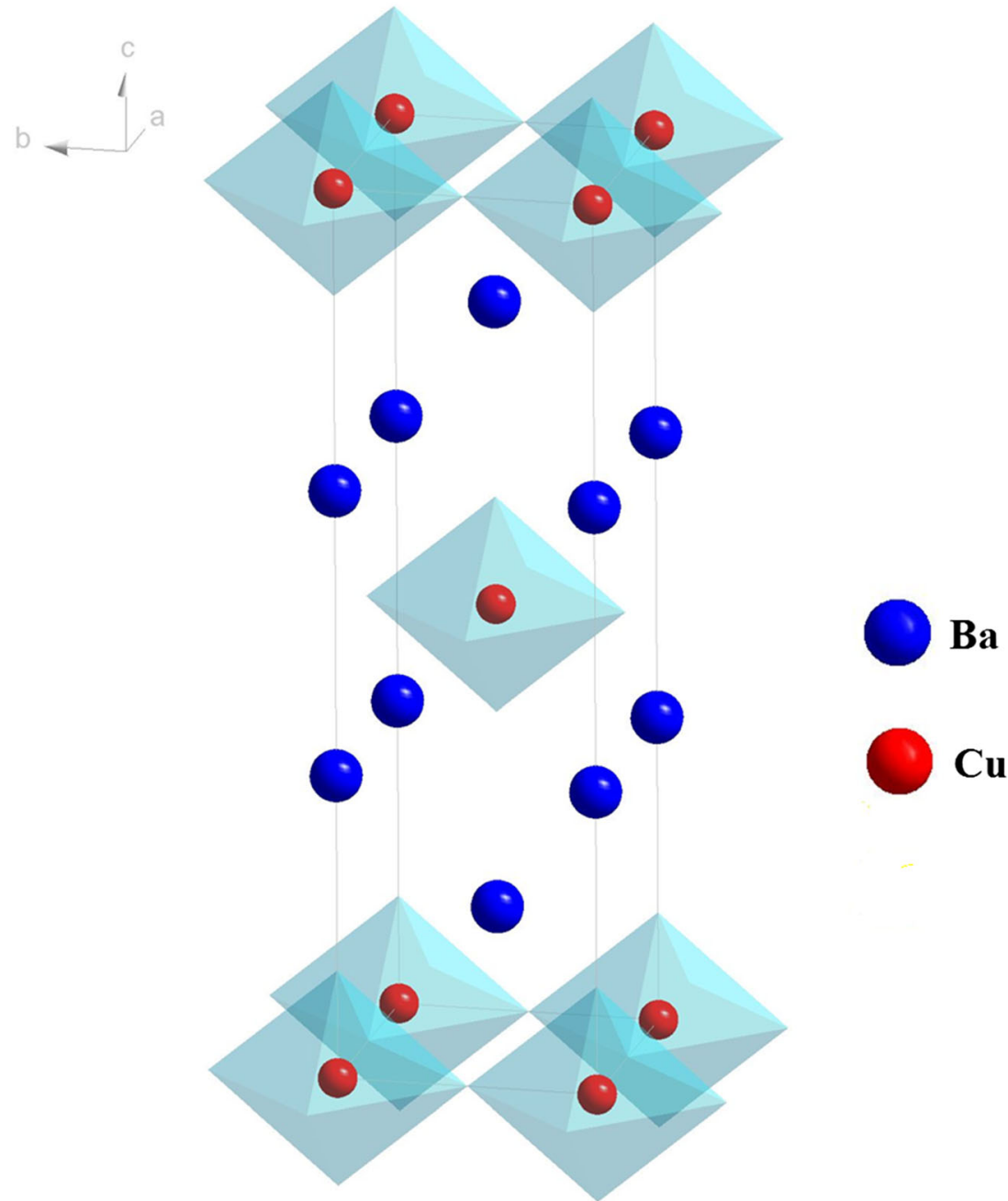

$[20,24,25]$. The hole-doped $\mathrm{Sr}_{2} \mathrm{CuO}_{3+\delta}$ superconductor crystallizes into an oxygen-deficient $\mathrm{La}_{2} \mathrm{CuO}_{4}$ (i.e., $\mathrm{K}_{2} \mathrm{NiF}_{4}$ ) structure. Our previous studies show that the maximum $T_{c}$ of $\mathrm{Sr}_{2} \mathrm{CuO}_{3+\delta}$ can reach $95 \mathrm{~K}$ with a nominal $\delta \sim 0.4(p \sim 0.8)$ which will be located in the very heavily overdoped region $[20,25]$. Further, $T_{c}{ }^{\max }=98 \mathrm{~K}$ is achieved in the Basubstituted material $\mathrm{Sr}_{2-x} \mathrm{Ba}_{x} \mathrm{CuO}_{3+\delta}(0 \leq x \leq 0.6)$ with $x=$ 0.6 that reaches the record value among the single-layer copper oxide superconductors - even higher than that of Hg1201 [26]. These materials are all synthesized at high oxygen pressure and characterized as heavily overdoping cuprates.

To deal with the topic, we have been focusing for long time on the alkaline earth and copper oxide system that is the simplest composition to build up cuprate superconductors $[18,20$, 25-29]. However, high pressure is usually required in order to get the structure by design. Recently, we discovered a new type of superconductor $\mathrm{Ba}_{2} \mathrm{CuO}_{4-y}$ (briefly coined $\mathrm{Ba} 214$ ) with the oxygen-deficient $\mathrm{La}_{2} \mathrm{CuO}_{4}$ ( $\left.\mathrm{La} 214\right)$ structure [18]. It has so far the only compressed local octahedron, in sharp contrast to the elongated octahedron for all previously known cuprate superconductors. Moreover, it is in an extremely overdoped state as well. The new superconductor shows superconductivity with $T_{c}$ up to $73 \mathrm{~K}$. It is generally believed that orbital reversal and extreme overdoping will suppress superconductivity in cuprates [11]. These suggest that Ba214 is a member of a new branch of high $T_{c}$ cuprate superconducting materials with unconventional features that challenge the established wisdom of HTS [30].

\section{Experiments}

The polycrystalline sample of $\mathrm{Ba} 214$ was synthesized at high pressure $(\sim 18 \mathrm{GPa})$ and high temperature $\left(\sim 1000^{\circ} \mathrm{C}\right)$ conditions with a highly self-oxidizing atmosphere [27-29] using a 6 over 8 double-stage type multianvil high-pressure facility. Phase purity of the obtained high-pressure products was examined by both powder X-ray diffraction (XRD) at room temperature and neutron at low temperature. Rietveld refinements were performed using the GSAS software package. The direct-current magnetic susceptibility was measured using a commercial SQUID-VSM (Quantum Design). Soft X-ray 

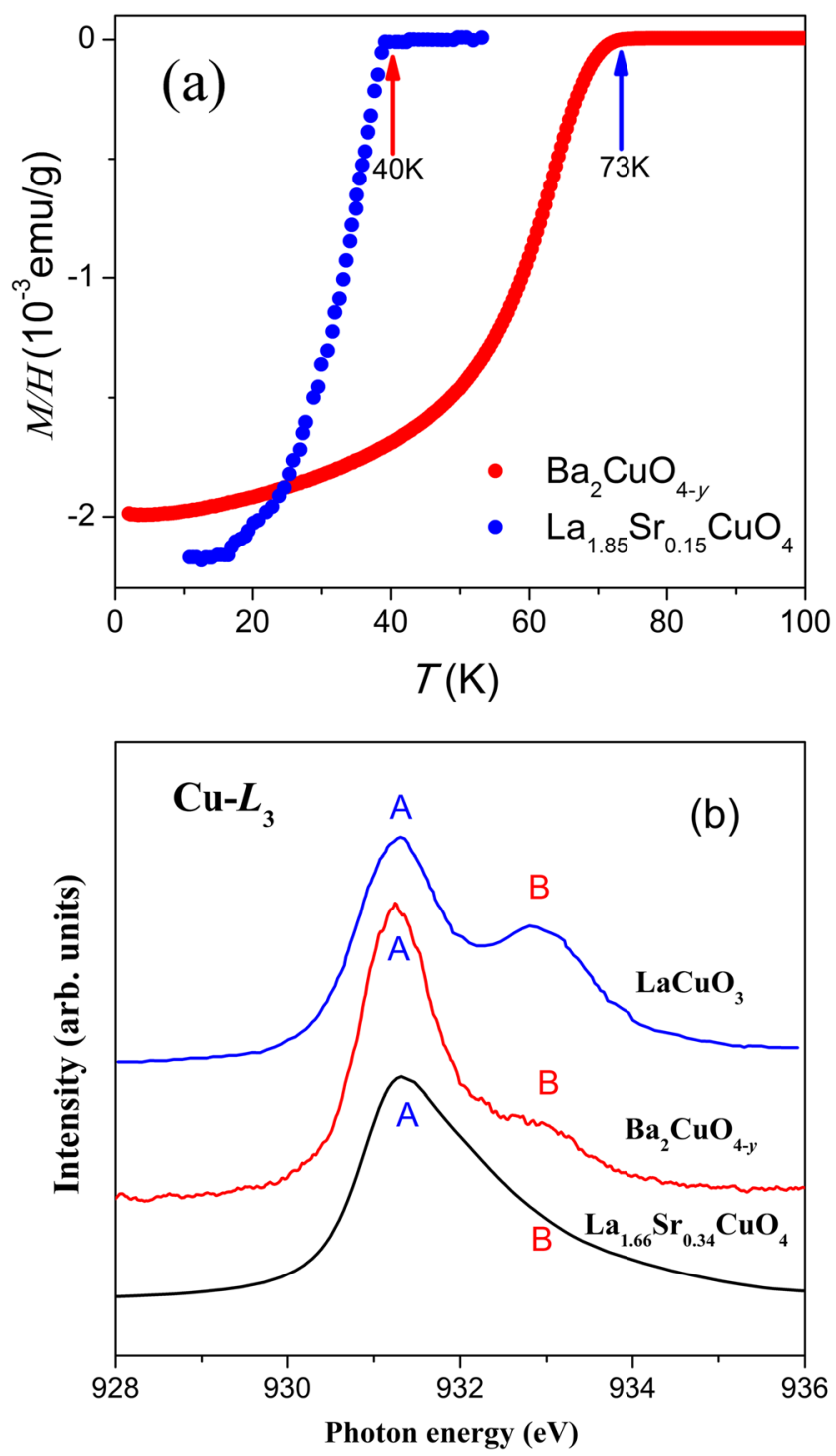

Fig. 2 a Temperature dependence of field-cooling (FC) $d c$ magnetic susceptibility of Ba214 and optimum doped $\mathrm{La}_{1.85} \mathrm{Sr}_{0.15} \mathrm{CuO}_{4}$ taken from ref. 32. b The $\mathrm{Cu}-L_{3}$ edge XAS spectrum of $\mathrm{Ba}_{2} \mathrm{CuO}_{4-\mathrm{y}}$, $\mathrm{La}_{1.66} \mathrm{Sr}_{0.34} \mathrm{CuO}_{4}$, and $\mathrm{LaCuO}_{3}$

absorption spectroscopy (XAS) at the $\mathrm{Cu} L_{2,3}$ edges and O-K edge was measured at beamline BL11A of the NSRRC in Taiwan.

\section{Results and Discussion}

High- $T_{c}$ superconducting Ba214 samples can be synthesized only in a narrow range of the nominal oxygen deficiency $y$ 0.8 . Powder $\mathrm{Ba} 214$ compound crystallizes into the $\mathrm{La}_{2} \mathrm{CuO}_{4}$ type structure with space group $I 4 / \mathrm{mmm}$ and gives rise to the lattice parameters $a=4.0030(3) \AA$ and $c=12.942(1) \AA$. The $\mathrm{Cu}-\mathrm{O}$ bond lengths for $\mathrm{Ba} 214$ are estimated to be 2.0015(2) $\AA$ in the plane and 1.861(8) $\AA$ along the $c$-axis (corresponding to the apical $\mathrm{O}$ distance $d_{A}$ ). It yields both records of the longest

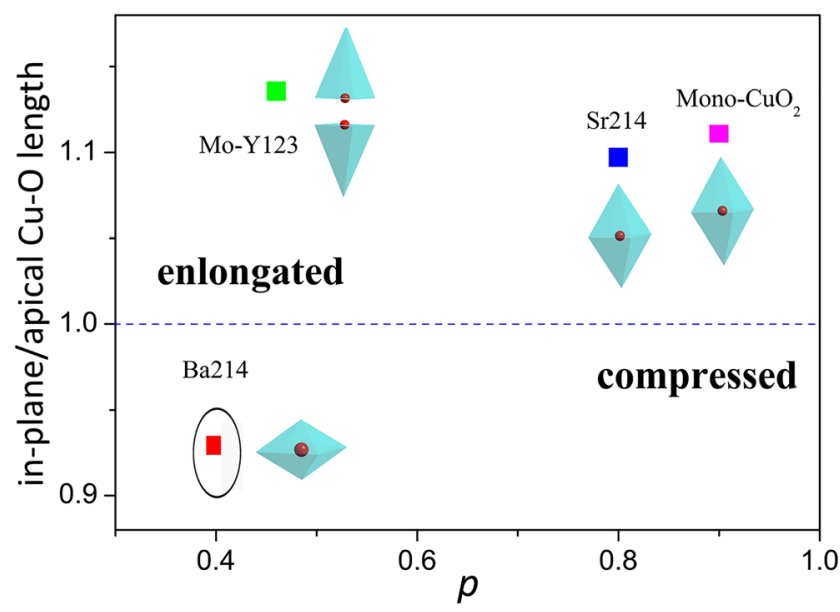

Fig. 3 The ratio between the bond lengths of in-plane $\mathrm{Cu}-\mathrm{O}$ and copper apical oxygen of heavily overdoped cuprates $\mathrm{Cu}_{0.575} \mathrm{Mo}_{0.25} \mathrm{Sr}_{2} \mathrm{YCu}_{2} \mathrm{O}_{7.54}$ (Mo-Y123), monolayer $\mathrm{CuO}_{2}\left(\mathrm{Mono}-\mathrm{CuO}_{2}\right)$, and $\mathrm{Sr}_{2} \mathrm{CuO}_{3+\delta}(\mathrm{Sr} 214)$, compared with $\mathrm{Ba} 214$

in-plane $\mathrm{Cu}-\mathrm{O}$ bond length and shortest apical-O distance among hole-doped cuprates as known so far. This may be attributed to the extremely large $\mathrm{A}$ site cation $\mathrm{Ba}^{2+}$ dramatically expanding the in-plane $\mathrm{Cu}-\mathrm{O}$ bond to the unprecedented length ever reported. As a consequence, the octahedron in turn becomes compressed instead of elongated as shown in Fig. 1. The locally compressed octahedron of Ba214 is exceptionally opposite to the previously "conventional" cuprates [31], which strongly suggests Ba214 may belong to a new type of "non-conventional" high- $T_{c}$ cuprate superconductor.

Temperature dependence of field-cooling (FC) $d c$ magnetic susceptibility of the Ba214 compound is shown in Fig. 2a together with optimum doped isostructural $\mathrm{La}_{1.85} \mathrm{Sr}_{0.15} \mathrm{CuO}_{4}$ taken from ref. 32 . The Ba214 sample shows a $T_{c}^{\max } \sim 73 \mathrm{~K}$ after post annealing under $\mathrm{O}_{2}$ flow. The bulk superconductivity behavior of the Ba214 sample was also confirmed by the muon-spin-rotation ( $\mu \mathrm{SR})$ and the specific heat measurements.

$\mathrm{X}$-Ray absorption spectroscopy (XAS) is a very useful tool to detect the doped hole numbers and the distribution in cuprate superconductors [33]. The result obtained from both the study of the $\mathrm{Cu}-L_{3}$ edge and $\mathrm{O}-\mathrm{K}$ edge spectrum indicated a very high doping level in the Ba214 sample, consistent with the estimated $\delta$ values and the XRD spectrum Rietveld refinements. We take the bulk-sensitive fluorescence-yield (FY) mode measurement results. Figure $2 \mathrm{~b}$ shows the $\mathrm{Cu}-L_{3}$ spectra of $\mathrm{Ba}_{2} \mathrm{CuO}_{4-y}$ together with spectra for the overdoped $\mathrm{La}_{1.66} \mathrm{Sr}_{0.34} \mathrm{CuO}_{4}$ [33] and perovskite $\mathrm{LaCuO}_{3}$ [34] as references. Two peaks are observed in Ba214. Peak A around $931.3 \mathrm{eV}$, assigned to a $2 p^{5} 3 d^{10}$ final state (upper Hubbard band) coming from a $2 p^{6} 3 d^{9}$ initial state, and peak B at the high-energy side of peak $\mathrm{A}$, around $932.9 \mathrm{eV}$, are assigned to a $2 p^{5} 3 d^{10} L$ ( $L$ refers to a hole in the $\mathrm{O} 2 p$ ligand state) coming from a $2 p^{6} 3 d^{9} L$ initial state and strongly related to the $\mathrm{Cu}^{3+}$ state. In the $\mathrm{Cu}-L_{3}$ XAS spectrum, the intensity of B peak for 
$\mathrm{Ba}_{2} \mathrm{CuO}_{4-\mathrm{y}}$ is stronger than that for highly overdoped $\mathrm{La}_{1.66} \mathrm{Sr}_{0.34} \mathrm{CuO}_{4}$ even if it is still weaker than that for "all$\mathrm{Cu}^{3+} \mathrm{LaCuO}_{3}(p=1)$ while they all have a nearly $180^{\circ} \mathrm{Cu}-$ $\mathrm{O}-\mathrm{Cu}$ bond with bond angle. The result explicitly indicates a very high doping level in Ba214, consistent with the estimated $\delta$ values.

In copper oxides, the octahedrons are generically elongated due to both Jahn-Teller effect and strong interlayer Coulomb interactions which push the $3 d x^{2}-y^{2}$ orbital topmost and the doped holes to reside primarily on the $3 d x^{2}-y^{2}$ orbital, forming the so-called Zhang-Rice (Z-R) singlet [35] via strong hybridization with oxygen $2 p$ orbital. It is very fascinating that the overdoped cuprates synthesized at high-pressure and highoxidizing atmosphere as mentioned before usually possess a short apical oxygen distance $d_{A}$ compared with the normal or "conventional" cuprates (the typical value of $2.42 \AA$ in $\left.\mathrm{La}_{2} \mathrm{CuO}_{4}\right): d_{A}=2.165 \AA$ for double-layered $\mathrm{Cu}_{0.575} \mathrm{Mo}_{0.25} \mathrm{Sr}_{2} \mathrm{YCu}_{2} \mathrm{O}_{7.54}(\mathrm{Mo}-\mathrm{Y} 123), d_{A}=2.11 \AA$ for monolayer $\mathrm{CuO}_{2}\left(\mathrm{Mono}-\mathrm{CuO}_{2}\right)$, and $d_{A}=2.085 \AA$ for $\mathrm{Sr}_{2} \mathrm{CuO}_{3+\delta}(\mathrm{Sr} 214)$, respectively [19, 20, 22]. Figure 3 shows the ratio of the bond lengths of in-plane $\mathrm{Cu}-\mathrm{O}$ and copper apical oxygen of Mo-Y123 (double layered), Mono- $\mathrm{CuO}_{2}$, and Sr214, compared with Ba214. Nonetheless, the octahedrons (pyramid for Mo-Y123) of all above non-conventionallike cuprates are still elongated which result in an ordinary degenerate of $\mathrm{Cu} e_{\mathrm{g}}$ orbitals split into a higher $3 d x^{2}-y^{2}$ orbital vs a lower $3 d z^{2}-r^{2}$ orbital except Ba214.

The Ba214 sample possesses a compressed octahedron which is completely different from the conventional cuprates. In a compressed octahedron, the $\mathrm{Cu} 3 d 3 z^{2}-r^{2}$ orbital will be pushed higher than the Cu3d $x^{2}-y^{2}$ orbital level. This suggests that the doped holes may reside preferentially in the $3 d z^{2}-r^{2}$ orbital and considerable $d 3 z^{2}-r^{2}$ orbital character might be mixed up in the states near the Fermi level that has been thought to be harmful for high$T_{c}$ superconductivity. It also means pairing symmetry of Ba214 may carry admixtures from more than one gap, and the system becomes more three-dimensional. It renders this new HTS cuprate a multi-band system like the iron-based superconductors [36-38]. K. Liu et al. suggested that the AFM fluctuations in onedimensional $\mathrm{Cu}-\mathrm{O}$ chains may play an important role in the superconducting pairing of electrons [36]. T. Maier et al. found two domes of pairing strength while one corresponds to the extremely overdoped regime and there is significant pairing strength in both the $d$-wave and $s^{ \pm}$channels in the overdoped regime [37]. M. Jiang et al. provided a new model featuring in a $\mathrm{Cu}-3 d$ multiplet structure that only the ligand $\mathrm{O}-2 p$ orbitals play an essential role [38].

The unique structure features of compressed octahedron in extremely overdoped $\mathrm{Ba}_{2} \mathrm{CuO}_{4-y}$ with conventional constitution of alkaline earth copper oxides kindled keen interest in a deeper understanding of the unconventional copper oxide superconductor.
Funding information The work was supported by Ministry of Science and Technology(MOST) and Natural Science Foundation (NSF) of China, Max Planck Institute (MPI), US NSF, and US Department of Energy, Bureau of Energy Sciences.

Open Access This article is distributed under the terms of the Creative Commons Attribution 4.0 International License (http:// creativecommons.org/licenses/by/4.0/), which permits unrestricted use, distribution, and reproduction in any medium, provided you give appropriate credit to the original author(s) and the source, provide a link to the Creative Commons license, and indicate if changes were made.

\section{References}

1. Geballe, T.H.: Paths to higher temperature superconductors. Science. 259, 1550 (1993)

2. Bednorz, J.G., Müller, K.A.: Possible high $T_{c}$ superconductivity in the Ba-La-Cu-O system. Z. Phys. B. Condens. Matter. 64, 189-193 (1986)

3. Wu, M.K., et al.: Superconductivity at $93 \mathrm{~K}$ in a new mixed-phase $\mathrm{Yb}-\mathrm{Ba}-\mathrm{Cu}-\mathrm{O}$ compound system at ambient pressure. Phys. Rev. Lett. 58, 908-910 (1987)

4. Zhao, Z.X., et al.: High $T_{c}$ superconductivity of $\mathrm{Sr}(\mathrm{Ba})-\mathrm{La}-\mathrm{Cu}$ oxides. Chin. Sci. Bull. 8, 522-525 (1987)

5. Logvenov, G., Gozar, A., Bozovic, I.: High-temperature superconductivity in a single copper-oxygen plane. Science. 326, 699-702 (2009)

6. Geballe, T.H.: The never-ending search for high-temperature superconductivity. J. Supercond. Nov. Magn. 19, 261-276 (2006)

7. Kivelson, S.A., Lederer, S.: Linking the pseudogap in the cuprates with local symmetry breaking: a commentary. Proc. Natl. Acad. Sci. 116, 14395-14397 (2019)

8. Geballe, T.H., Hammond, R.H., Wu, P.M.: What $T_{c}$ tells. Phys. C. 514, 9-16 (2015)

9. Jin, C.Q., et al.: Superconductivity at $80 \mathrm{~K}$ in $(\mathrm{Sr}, \mathrm{Ca})_{3} \mathrm{Cu}_{2} \mathrm{O}_{2+\delta} \mathrm{Cl}_{2-\mathrm{y}}$ induced by apical oxygen doping. Nature. 375, 301 (1995)

10. Zhang, H., Sato, H.: Universal relationship between $T_{c}$ and the hole content in p-type cuprate superconductors. Phys. Rev. Lett. 70, 1697 (1993)

11. Keimer, B., Kivelson, S.A., Norman, M.R., Uchida, S., Zaanen, J.: From quantum matter to high-temperature superconductivity in copper oxides. Nature. 518, 179-186 (2015)

12. Lee, P.A., Nagaosa, N., Wen, X.G.: Doping a Mott insulator: physics of high-temperature superconductivity. Rev. Mod. Phys. 78, 1785 (2006)

13. Scalapino, D.J.: A common thread: the pairing interactions for unconventional superconductors. Rev. Mod. Phys. 84, 1383-1417 (2012)

14. Emery, V.J., Kivelson, S.A.: Importance of phase fluctuations in superconductors with small superfluid density. Nature. 374, 434 (1995)

15. Le Tacon, M., et al.: Dispersive spin excitations in highly overdoped cuprates revealed by resonant inelastic X-ray scattering. Phys. Rev. B. 88, 020501 (2013)

16. Bozovic, I., He, X., Wu, J., Bollinger, A.T.: Dependence of the critical temperature in overdoped copper oxides on superfluid density. Nature. 536, 309-311 (2016)

17. Geballe, T.H., Marezio, M.: Enhanced superconductivity in $\mathrm{Sr}_{2} \mathrm{CuO}_{4-\mathrm{v}}$. Phys. C. 469, 680 (2009)

18. Li, W.M., Zhao, J.F., Cao, L.P., Hu, Z., Huang, Q.Z., Wang, X.C., Liu, Y., Zhao, G.Q., Zhang, J., Liu, Q.Q., Yu, R.Z., Long, Y.W., Wu, H., Lin, H.J., Chen, C.T., Li, Z., Gong, Z.Z., Guguchia, Z., 
Kim, J.S., Stewart, G.R., Uemura, Y.J., Uchida, S., Jin, C.Q.: Superconductivity in a unique type of copper oxide. Proc. Natl. Acad. Sci. 116, 12156-12160 (2019)

19. Gauzzi, A., et al.: Bulk superconductivity at $84 \mathrm{~K}$ in the strongly overdoped regime of cuprates. Phys. Rev. B. 94, 180509 (2016)

20. Liu, Q.Q., Yang, H., Qin, X.X., Yu, Y., Yang, L.X., Li, F.Y., Yu, R.C., Jin, C.Q., Uchida, S.: Enhancement of the superconducting critical temperature of $\mathrm{Sr}_{2} \mathrm{CuO}_{3+\delta}$ up to $95 \mathrm{~K}$ by ordering dopant atoms. Phys. Rev. B. 74, 100506 (R) (2006)

21. Ono, A.: High-pressure synthesis of Mo-containing 1212 and 1222 compounds, $(\mathrm{Cu}, \mathrm{Mo}) \mathrm{Sr}_{2} \mathrm{YCu}_{2} \mathrm{O}_{\mathrm{z}}$ and $(\mathrm{Cu}, \mathrm{Mo}) \mathrm{Sr}_{2}(\mathrm{Y}, \mathrm{Ce})_{2} \mathrm{Cu}_{2} \mathrm{O}_{\mathrm{z}}$. Jpn. J. Appl. Phys. 32, 4517 (1993)

22. Zhong, Y., et al.: Nodeless pairing in superconducting copper-oxide monolayer films on $\mathrm{Bi}_{2} \mathrm{Sr}_{2} \mathrm{CaCu}_{2} \mathrm{O}_{8+\delta}$. Sci. Bull. 61, 1239-1247 (2016)

23. Zhu, G.Y., Wang, Z.Q., Zhang, G.M.: Two-dimensional topological superconducting phases emerged from $d$-wave superconductors in proximity to antiferromagnets. EPL. 118, 37004 (2017)

24. Hiroi, Z., Takano, M., Azuma, M., Takeda, Y.: A new family of copper oxide superconductors $\mathrm{Sr}_{\mathrm{n}+1} \mathrm{Cu}_{\mathrm{n}} \mathrm{O}_{2 \mathrm{n}+1+\delta}$ stabilized at high pressure. Nature. 364(375), 315 (1993)

25. Yang, H., Liu, Q.Q., Li, F.Y., Jin, C.Q., Yu, R.C.: Structure and microstructure of superconductor $\mathrm{Sr}_{2} \mathrm{CuO}_{3+\delta}$ (nominal $\delta=0.4$ ) prepared under high pressure. Supercond. Sci. Technol. 19, 934-940 (2006)

26. Gao, W.B., Liu, Q.Q., Yang, L.X., Yu, Y., Li, F.Y., Jin, C.Q., Uchida, S.: Out-of-plane effect on the superconductivity of $\mathrm{Sr}_{2}$ ${ }_{-\mathrm{x}} \mathrm{Ba}_{\mathrm{x}} \mathrm{CuO}_{3+\delta}$ with $T_{c}$ up to $98 \mathrm{~K}$. Phys. Rev. B. 80, 94523 (2009)

27. Jin, C.Q.: Using pressure effects to create new emergent materials by design. MRS Adv. 2, 2587 (2017)

28. Jin, C.Q., Adachi, S., Wu, X.J., Yamauchi, H.: A new superconducting homologous series of compounds: $\mathrm{Cu}-12(\mathrm{n}-1) \mathrm{n}$. Advances in superconductivity VII, p. P249. Springer-Verlag, Tokyo (1995)
29. Jin, C.Q., et al.: $117 \mathrm{~K}$ superconductivity in the $\mathrm{Ba}-\mathrm{Ca}-\mathrm{Cu}-\mathrm{O}$ system. Physica C. 223, 238 (1994)

30. Scalapino, D.J.: A different branch of the high $T_{c}$ family? Proc. Natl. Acad. Sci. 116, 12129-12130 (2019)

31. Huang, Q., Lynn, J.W., Xiong, Q., Chu, C.W.: Oxygen dependence of the crystal structure of $\mathrm{HgBa}_{2} \mathrm{CuO}_{4+\delta}$ and its relation to superconductivity. Phys. Rev. B. 52, 462-470 (1995)

32. Tarascon, J.M., Greene, L.H., Mckinnon, W.R., Hull, G.W., Geballe, T.H.: Superconductivity at $40 \mathrm{~K}$ in the oxygen-defect perovskites $\mathrm{La}_{2-\mathrm{x}} \mathrm{Sr}_{\mathrm{x}} \mathrm{CuO}_{4-y}$. Science. 235, 1373-1376 (1987)

33. Chen, C.T., Tjeng, L.H., Kwo, J., Kao, H.L., Rudolf, P., Sette, F., Fleming, R.M.: Out-of-plane orbital characters of intrinsic and doped holes in $\mathrm{La}_{2-x} \mathrm{Sr}_{x} \mathrm{CuO}_{4}$. Phys. Rev. Lett. 68, 2543-2546 (1992)

34. Mizokawa, T., Fujimori, A., Namatame, H., Takeda, Y., Takano, M.: Electronic structure of tetragonal $\mathrm{LaCuO}_{3}$ studied by photoemission and X-ray absorption spectroscopy. Phys. Rev. B. 57, 9550-9556 (1998)

35. Zhang, F.C., Rice, T.M.: Effective Hamiltonian for the superconducting Cu oxides. Phys. Rev. B. 37, 3759-3761 (1988)

36. Liu, K., Lu, Z.Y., Xiang, T.: Electronic structures of quasi-onedimensional cuprate superconductors $\mathrm{Ba}_{2} \mathrm{CuO}_{3+\delta}$. Phys. Rev. M. 3, 044802 (2019)

37. Maier, T., Berlijn, T., Scalapino, D.J.: Two pairing domes as $\mathrm{Cu}^{2+}$ varies to $\mathrm{Cu}^{3+}$. Phys. Rev. B. 99, 224515 (2019)

38. Jiang, M., Moeller, M., Berciu, M., Sawatzky, G. A.: Relevance of $\mathrm{Cu}-3 \mathrm{~d}$ multiplet structure in models of high $T_{c}$ cuprates. arXiv: 1906.10254v2 (2019)

Publisher's note Springer Nature remains neutral with regard to jurisdictional claims in published maps and institutional affiliations. 